Doi: $10.15863 /$ TAS

International Scientific Journal Theoretical \& Applied Science

p-ISSN: 2308-4944 (print) e-ISSN: 2409-0085 (online)

Year: $2015 \quad$ Issue: 02 Volume: 22

Published: $28.02 .2015 \quad$ http://www.T-Science.org
Denis Alexandrovich Chemezov

Master of Engineering and

Technology, Corresponding member of International

Academy TAS, Lecturer of

Vladimir Industrial College,

Russia

chemezov-da@yandex.ru

SECTION 7. Mechanics and machine construction.

\title{
DEFORMATIONS OF THE CONTACT SURFACES OF THE PARTS WHEN PRESSING-IN
}

Abstract: The article presents the results of the implementation of computer simulation of the process of pressing the shaft into the bushing: the stress values according to von Mises, maximum strain, pressure, the resultant displacement, the resultant velocity and resultant acceleration of deformation of the parts. See the text of the decision task $k$-file specifying the material properties of parts, the number of nodes and elements of the models, boundary conditions, time of simulation and other necessary information.

Key words: bushing, shaft, pressing-in, strain, stress.

Language: Russian

Citation: Chemezov DA (2015) DEFORMATIONS OF THE CONTACT SURFACES OF THE PARTS WHEN PRESSING-IN. ISJ Theoretical \& Applied Science 02 (22): 1-7. http://dx.doi.org/10.15863/TAS.2015.02.22.1

\section{УДК 621.713.24}

\section{ДЕФОРМАЦИИ КОНТАКТНЫХ ПОВЕРХНОСТЕЙ ДЕТАЛЕЙ ПРИ ЗАПРЕССОВЫВАНИИ}

Аннотация: В статье представлены результаты реализации компьютерного имитационного моделирования проиесса запрессовывания вала во втулку: значения напряжения по фон Мизесу, максимальной деформации, давления, результирующего перемещчения, результирующей скорости и результирующего ускорения деформации деталей. Приведен текст решения задачи (k-файл) с указанием свойств материалов деталей, количества узлов и элементов моделей, граничных условий, времени моделирования и другой необходимой информации.

Ключевые слова: втулка, вал, запрессовывание, деформация, напряжение.

На производстве, детали машин соединяются различными способами. Неразъемное соединение деталей с гарантированным натягом собирается путем запрессовывания вала (цилиндр с постоянным или переменным диаметром) во втулку (деталь с отверстием постоянного или переменного диаметра) [1]. Натяг между контактными поверхностями деталей создается до сборки и будет равен разности значений посадочных размеров вала и втулки [2].

Процесс запрессовывания сопровождается деформацией контактных поверхностей вала и втулки. В результате упругой деформации, возникают концентрированные напряжения материалов деталей в местах сопряжения. Происходит «срезание» слоев материалов вала и втулки. Наружный диаметр запрессованного вала будет равен внутреннему диаметру втулки. Неподвижность прессового соединения, при воздействии статических и динамических нагрузок, обеспечивается за счет сил трения на контактных поверхностях деталей [3].

Величина деформации и соответственно напряжения материалов деталей будет зависеть от ряда факторов (свойств материала, величины натяга, способа запрессовывания и др.). Определение значений этих величин позволяет сделать прогноз надежности неразъемного соединения.

Процесс запрессовывания вала во втулку моделировался в программной среде LS-DYNA. Для решения задачи в компьютерной программе были построены объемные твердотельные 
модели деталей (LS-PREPOST $\rightarrow$ Blockm interface $\rightarrow$ Butterfly blocks) [4]. Численное значение заданных размеров моделей:

- втулки: наружный диаметр - 20 мм, внутренний диаметр - 10 мм, длина - 20 мм;

- вала: наружный диаметр - 10,08 мм, длина - 20 мм.

Втулка фиксировалась и не имела линейных перемещений по координатным осям $\mathrm{X}, \mathrm{Y}, \mathrm{Z}$ и вращательного движения вокруг своей оси. Ось вала совмещалась с осью втулки. Вал перемещался в одном направлении вдоль оси втулки. Принятый временной диапазон моделирования процесса - 0..0,405 сек. Все условия решаемой задачи записывались в текстовом файле в буквенно-цифровом виде [5]. Сокращенный вариант постановки задачи представлен ниже (знак \$ - комментарий).

\section{*KEYWORD}

*TITLE

Interference fit

*SECTION SOLID

1,1

\$ Material definitions

*MAT PLASTIC KINEMATIC

$\$$ mid ro e pr sigy etan beta

$1,2.7 \mathrm{e}-6,70,0.34,0.267,0.32,0$

$\$$ src srp fs vp

$0,0,0,0$

*PART

Solid 1

$\$$ pid secid mid eosid hgid grav adpopt tmid

$1,1,1,0,0,0,0,0$

*PART

Solid 2

$\$$ pid secid mid eosid hgid grav adpopt tmid

$2,1,1,0,0,0,0,0$

$\$$ Element definitions

*ELEMENT SOLID

$\$$ eid pid n1 n2 n3 n4 n5 n6 n7 n8

$1,1,1,78,89,12,2,79,90,13$

$2,1,2,79,90,13,3,80,91,14$

$3,1,3,80,91,14,4,81,92,15$

$4,1,4,81,92,15,5,82,93,16$

$5,1,5,82,93,16,6,83,94,17$

$6,1,6,83,94,17,7,84,95,18$

$7,1,7,84,95,18,8,85,96,19$

$8,1,8,85,96,19,9,86,97,20$

$9,1,9,86,97,20,10,87,98,21$

$10,1,10,87,98,21,11,88,99,22$

47632, 2, 34264, 43372, 34220, 43339, 34263, 43371, 34219,43338

47633, 2, 34263, 43371, 34219, 43338, 34262, 43370, 34218,43337

47634, 2, 34262, 43370, 34218, 43337, 34261, 43369, 34217,43336

$47635,2,34261,43369,34217,43336,34260,43368$, 34216,43335

$47636,2,34260,43368,34216,43335,34259,43367$, 34215,43334
47637, 2, 34259, 43367, 34215, 43334, 34258, 43366, 34214,43333

$47638,2,34258,43366,34214,43333,34257,43365$, 34213,43332

$47639,2,34257,43365,34213,43332,34256,43364$, 34212,43331

47640, 2, 34256, 43364, 34212, 43331, 34255, 43363, 34211,43330

$\$$ Node definitions

*NODE

$\$$ nid $x$ y $z$ tc rc

$1,-7.07106781,-7.07106781,0,7,0$

$2,-7.07106781,-7.07106781,1.99999988,7,0$

$3,-7.07106781,-7.07106781,3.99999976,7,0$

$4,-7.07106781,-7.07106781,5.99999952,7,0$

$5,-7.07106781,-7.07106781,7.99999952,7,0$

$6,-7.07106781,-7.07106781,10,7,0$

$7,-7.07106781,-7.07106781,11.99999905,7,0$

$8,-7.07106781,-7.07106781,14,7,0$

$9,-7.07106781,-7.07106781,15.99999905,7,0$

$10,-7.07106781,-7.07106781,18,7,0$

$52804,2.05215836,4.5317963 \mathrm{e}-9,26.10000038,0,0$ $52805,2.05215836,4.5317963 \mathrm{e}-9,28.10000038,0,0$ 52806, 2.05215836, 4.5317963e-9, 30.10000038, 0, 0 52807, 2.05215836, 4.5317963e-9, 32.09999847, 0, 0 $52808,2.05215836,4.5317963 \mathrm{e}-9,34.09999847,0,0$ 52809, 2.05215836, 4.5317963e-9, 36.09999847, 0, 0 $52810,2.05215836,4.5317963 \mathrm{e}-9,38.09999847,0,0$ 52811, 2.05215836, 4.5317963e-9, 40.09999847, 0, 0 *INITIAL_VELOCITY_GENERATION

$\$$ nsid/pid styp omega vx vy vz

$2,2,1,0,0,-50$

$\$ \mathrm{xc}$ yc zc nx ny nz phase

$0,0,0,0,0,0,0$

*CONTACT_AUTOMATIC_SURFACE_TO_SURFAC $\mathrm{E}$

\$ ssid msid sstyp mstyp sboxid mboxid spr mpr

$1,2,3,3,0,0,0,0$

*CONTROL TERMINATION

$\$$ endtim endcyc dtmin endeng endmas

$0.405,0,0,0$

*DATABASE BINARY_D3PLOT

$\$$ dt lcdt beam npltc

$1.0 \mathrm{e}-3,0,0,0$

*CONTROL ENERGY

$\$$

*DATABASE GLSTAT

$1.0 \mathrm{e}-4$

*END

Имитация процесса запрессовывания деталей реализована в модуле LS-PREPOST. Напряженно-деформированное состояние материалов втулки и вала на 0,003, 0,15, 0,301 и 0,405 секундах процесса запрессовывания демонстрируют интерактивные эпюры (Fringe component) напряжения по фон Мизесу [6], максимальной (основной) деформации [7], результирующего ускорения деформации [8], давления (рис. $1-4,6-$ д). 


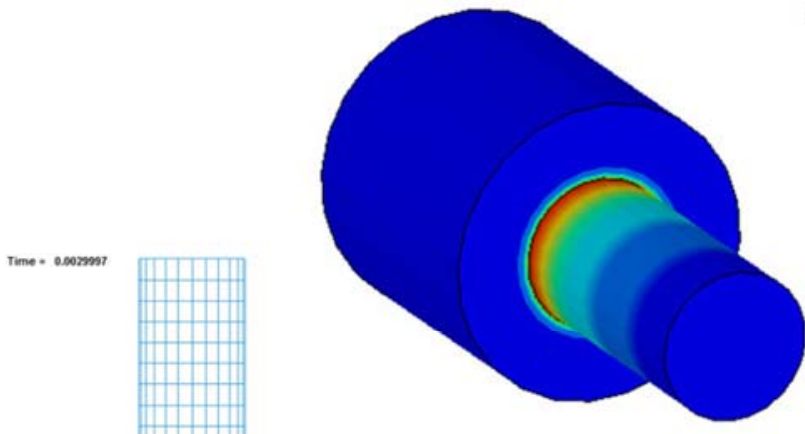

б)

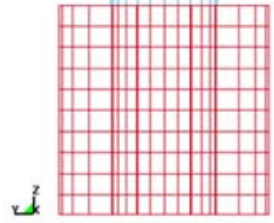

a)

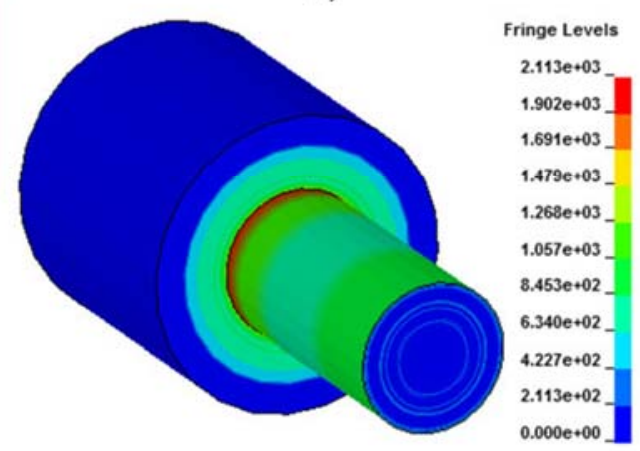

г)

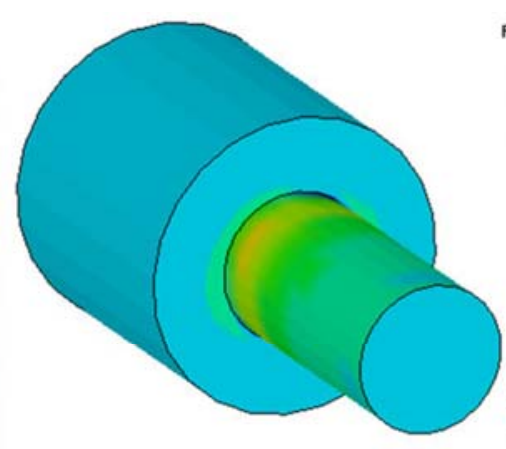
$2.934 \mathrm{e}-02$ $2.544 \mathrm{e}-02$ 2.154e-02 1.764e-02 $1.374 \mathrm{e}-02$ $9.837 \mathrm{e}-03$ $5.935 e-03$ $2.034 \mathrm{e}-03$ $1.868 \mathrm{e}-03$ $-5.769 \mathrm{e}-03$ B)

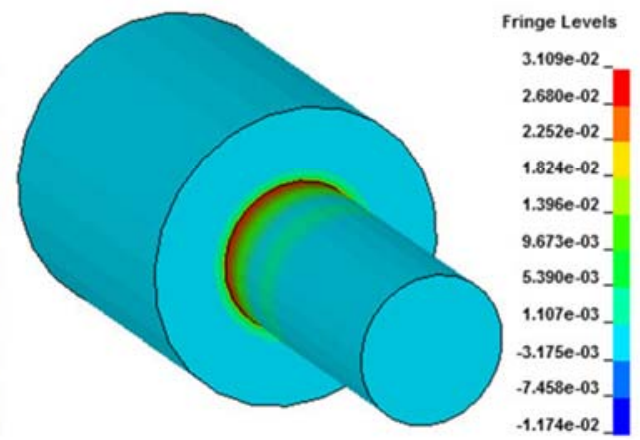

д)

Рисунок 1 - Напряженно-деформированное состояние втулки и вала на 0,003 сек. процесса запрессовывания: а - глубина запрессовывания вала во втулку; б - напряжение по фон Мизесу; в - максимальная деформация; г - результирующее ускорение деформации; д - давление.

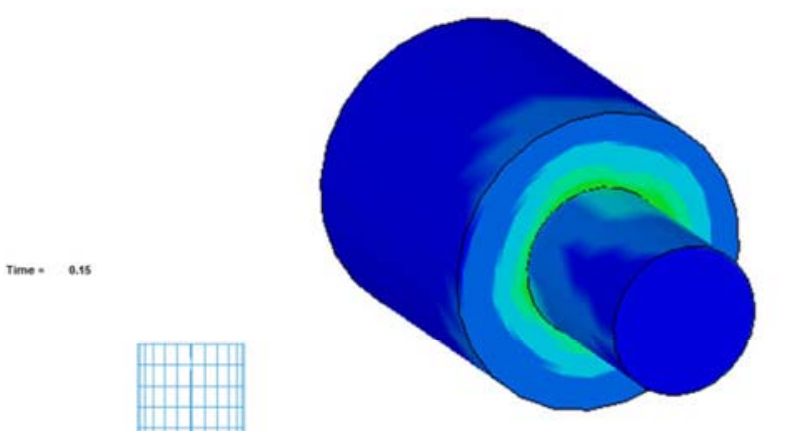

б)

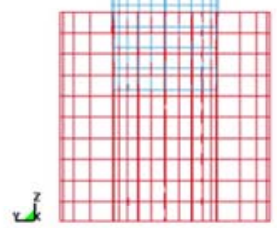

a)

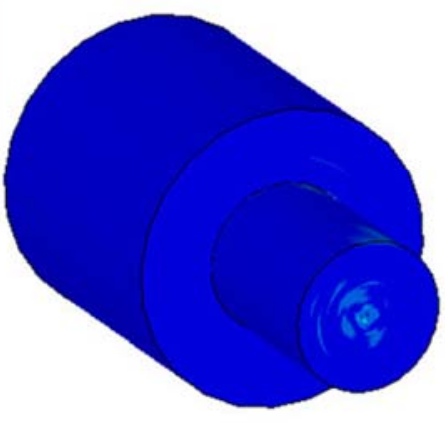

г)
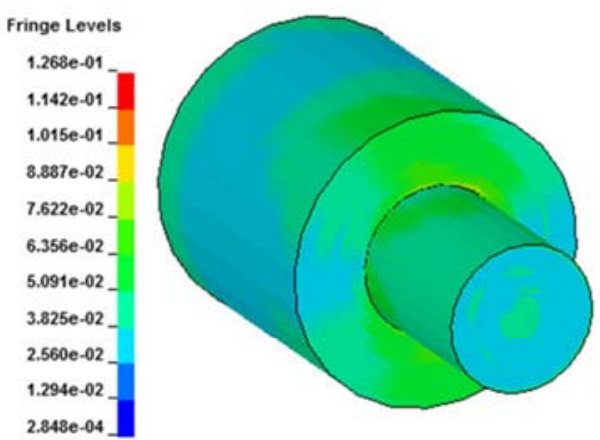

в)

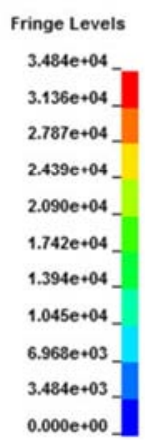

$0.000 \mathrm{e}+00$

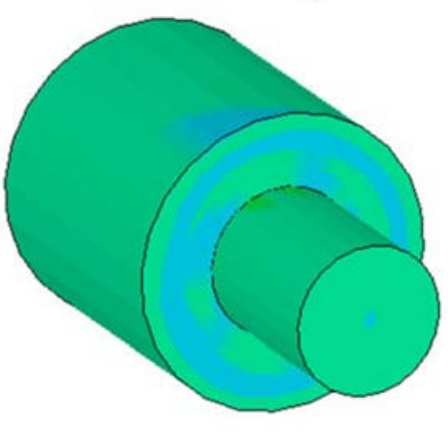

д)

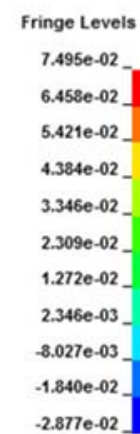

Fringe Levels $1.020 \mathrm{e}-01$ $8.673 \mathrm{e}-02$ $7.148 \mathrm{e}-02$ $5.624 \mathrm{e}-02$ 4.099e- 02 2.574e-02 $1.049 \mathrm{e}-02$ $-4.759 \mathrm{e}-03$ $-2.001 \mathrm{e}-02$ $-3.526 \mathrm{e}-02$ $-5.051 \mathrm{e}-02$

Рисунок 2 - Напряженно-деформированное состояние втулки и вала на 0,15 сек. процесса запрессовывания: а - глубина запрессовывания вала во втулку; б - напряжение по фон Мизесу; В - максимальная деформация; г - результирующее ускорение деформации; д - давление. 


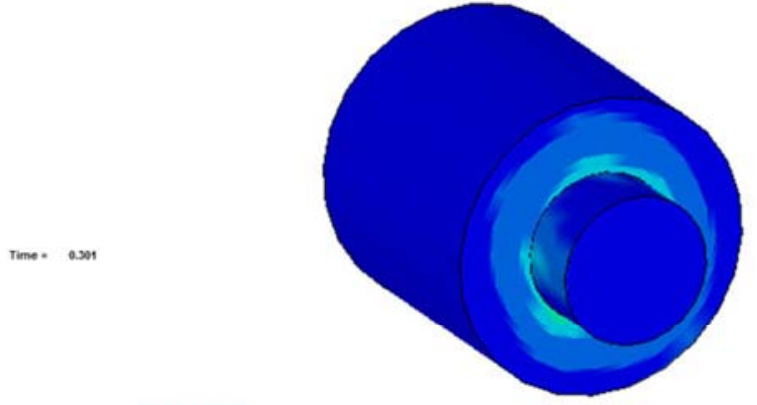

б)

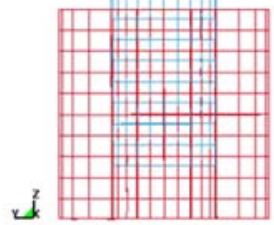

a)

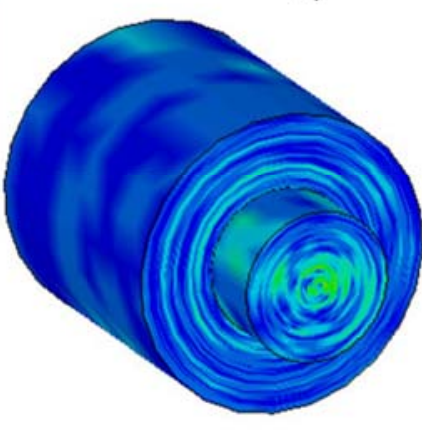

г)

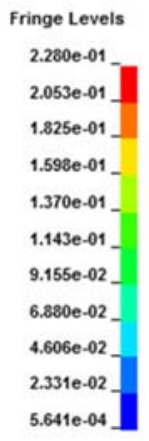

(2)

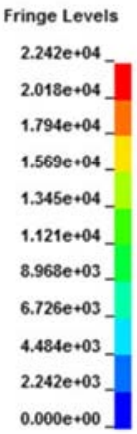

$0.000 \mathrm{e}+00]$

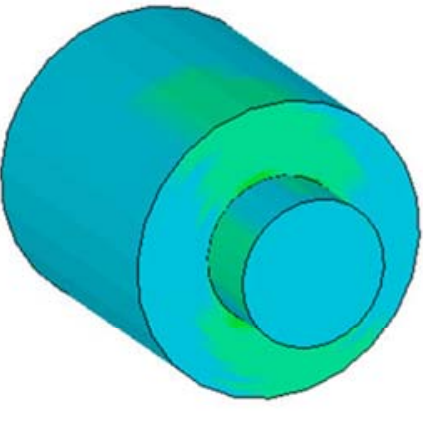

в)

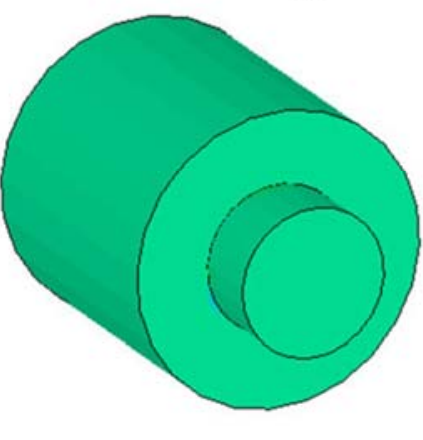

д)

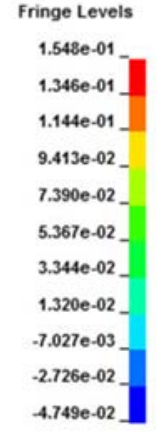

Fringe Leveis
$2.072 \mathrm{e}-01$
$1.751 \mathrm{e}-01$
$1.431 \mathrm{e}-01$
$1.110 \mathrm{e}-01$
$7.895 \mathrm{e}-02$
$4.689 \mathrm{e}-02$
$1.484 \mathrm{e}-02$
$-1.721 \mathrm{e}-02$
$-4.927 \mathrm{e}-02$
$-8.132 \mathrm{e}-02$
$-1.134 \mathrm{e}-01$

Рисунок 3 - Напряженно-деформированное состояние втулки и вала на 0,301 сек. процесса запрессовывания: а - глубина запрессовывания вала во втулку; б - напряжение по фон Мизесу; в - максимальная деформация; г - результирующее ускорение деформации; д - давление.

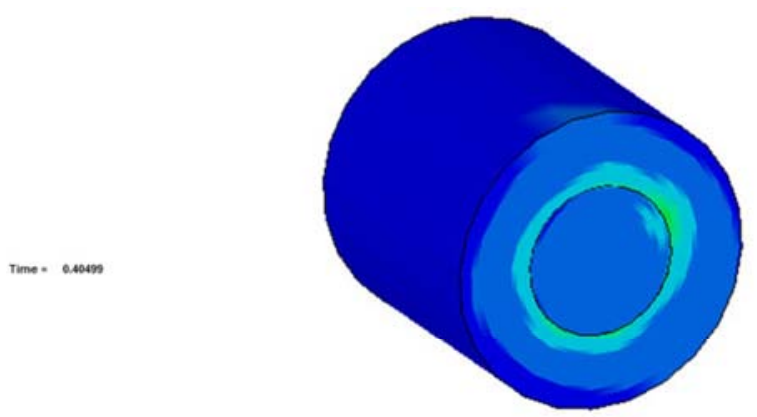

б)

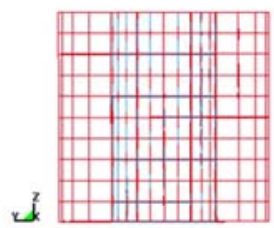

a)

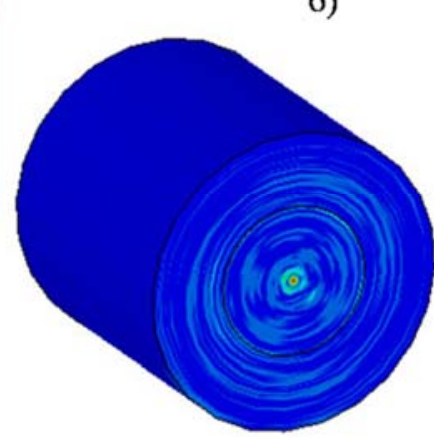

г)
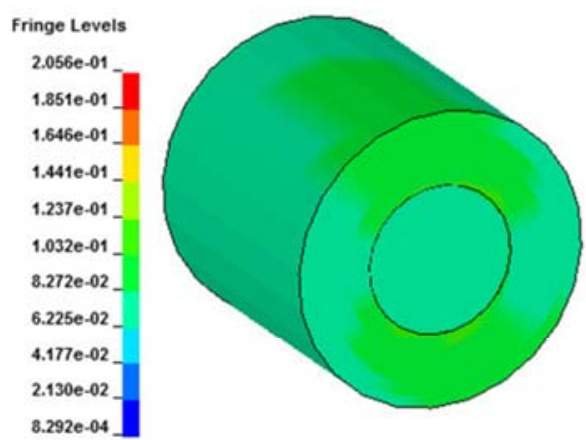

в)

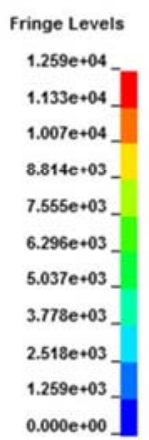

$0.000 \mathrm{e}+00$

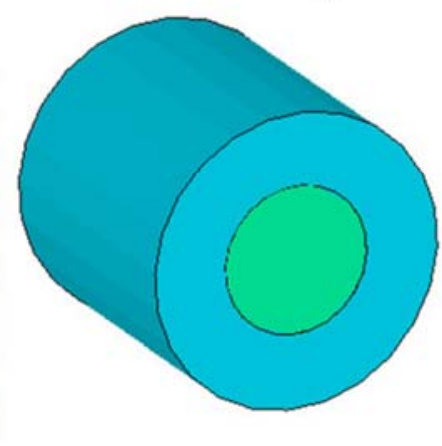

д)

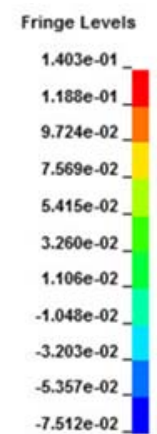

Fringe Levels 2.732e-01 $2.357 \mathrm{e}-01$ $1.981 \mathrm{e}-01$ $1.606 \mathrm{e}-01$ 1.231e-01_ $8.557 \mathrm{e}-02$ $4.805 e-02$ $1.052 \mathrm{e}-02$ $-2.700 \mathrm{e}-02$ $-6.453 \mathrm{e}-02$ $-1.021 \mathrm{e}-01$

Рисунок 4 - Напряженно-деформированное состояние втулки и вала на 0,405 сек. процесса запрессовывания: а - глубина запрессовывания вала во втулку; б - напряжение по фон Мизесу; в - максимальная деформация; г - результирующее ускорение деформации; д - давление. 
В момент захода вала под нагрузкой в отверстие, наибольшей деформации подвергаются поверхности непосредственно находящиеся в контакте. По мере перемещения вала, площадь соприкосновения поверхностей увеличивается, что приводит к увеличению интенсивности деформации материалов деталей. На глубине запрессовывания вала 8 мм (рис. 2, а) величина деформации увеличивается в 2 раза, напряжения - в 2,5 раза по сравнению со значениями в момент захода. На глубине запрессовывания вала 15 мм (рис. 3, а) деформация и напряжение материалов в местах контакта возрастают еще в 2 раза. На полной глубине запрессовывания 20 мм (рис. 4, а) отмечается некоторое уменьшение величины деформации и напряжения (остаточное) втулки и вала. Результирующее ускорение деформации наблюдается во всем объеме вала и втулки на 0,301 секунде процесса запрессовывания. Наибольшее значение ускорения деформации отображены на моделях цветовой гаммой по окружности, ближе к центру деталей.

С поверхности отверстия втулки (рис. 5), на всем временном диапазоне процесса запрессовывания, фиксировались показания результирующего перемещения детали [9] при нагрузке (рис. 6), напряжения [10] (рис. 7) и результирующей скорости деформации материала [11] (рис. 8).

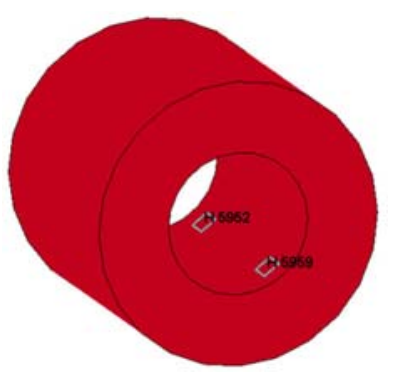

Рисунок 5 - Порядковые номера элементов модели втулки, с которых фиксировались показания.

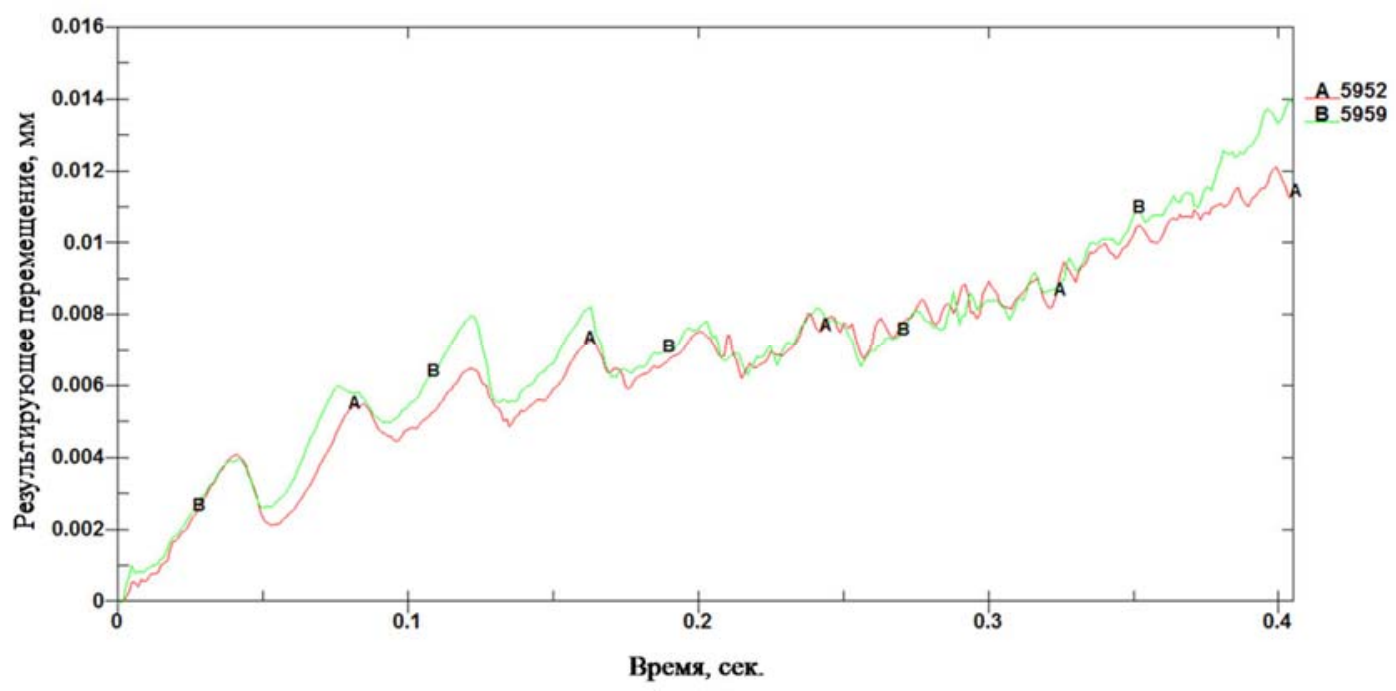

Рисунок 6 - Результаты отклика на внутренней поверхности втулки: зависимости результирующего перемещения детали при нагрузке от времени процесса запрессовывания. 


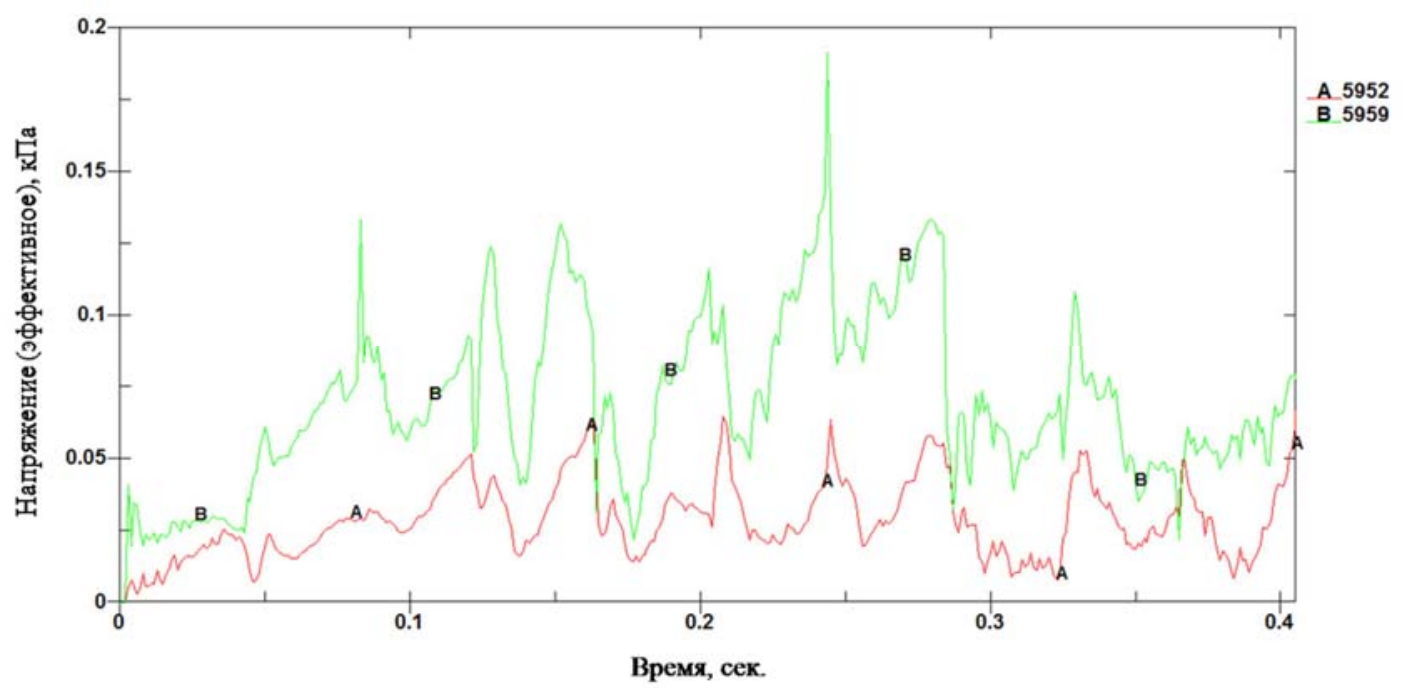

Рисунок 7 - Результаты отклика на внутренней поверхности втулки: зависимости напряжения материала втулки от времени процесса запрессовывания.

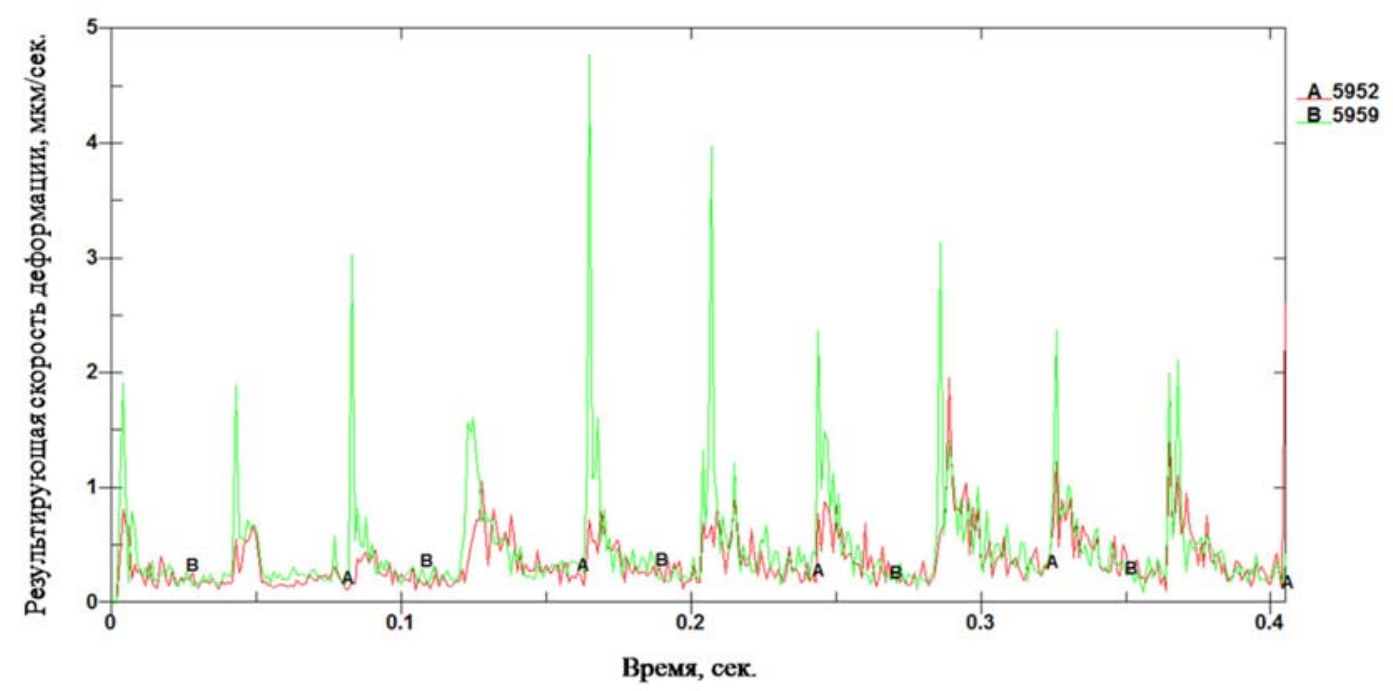

Рисунок 8 - Результаты отклика на внутренней поверхности втулки: зависимости результирующей скорости деформации материала втулки от времени процесса запрессовывания.

Зависимости, представленные на графиках, имеют выраженный скачкообразный характер изменения во времени. Наибольшее результирующее перемещение при нагрузке происходит на 0,405 сек. процесса и составляет со стороны запрессовывания вала 0,014 мм, с обратной стороны - 0,011 мм. Максимальное значение эффективного напряжения, возникающее в материале втулки, определено на 0,23 сек. процесса запрессовывания и составляет 0,19 кПа. Наибольшая результирующая скорость деформации материала втулки отмечена на 0,16 сек. На графике прослеживается цикл изменения скорости: фаза 1 , в которой величина параметра не выходит за пределы диапазона $0,2 \ldots 1,0$ мкм/сек. (длительность 0,05 сек.) и мгновенный скачок до максимального значения с последующим переходом в фазу 1 .

На основании полученных результатов имитирования явной динамики процесса запрессовывания вала во втулку можно сделать следующие выводы:

1. Положительные и отрицательные значения максимальных деформаций двух сопрягаемых деталей (см. цветовую шкалу справа от эпюры) определяют нарушение целостности при растяжении и сжатии слоев материалов;

2. Наибольшему напряжению подвергается материал втулки. Концентрация напряжения на торцевых поверхностях детали.

3. Более высокая интенсивность деформации и напряжения материала втулки 


$\begin{array}{llr}\text { Impact Factor ISRA }(\text { India) }=\mathbf{1 . 3 4 4} & \text { Impact Factor JIF } & =\mathbf{1 . 5 0 0} \\ \text { Impact Factor ISI (Dubai, UAE) }=\mathbf{0 . 8 2 9} & \text { Impact Factor GIF (Australia) }=\mathbf{0 . 3 5 6} \\ \text { based on International Citation Report }(\text { ICR) } & \text { Impact Factor SIS (USA) } & =\mathbf{0 . 4 3 8}\end{array}$

происходит на поверхности отверстия со стороны запрессовки (см. рис. 5 - 8, элемент Н 5959).

\section{References:}

1. (2015) Soedinenie s natyagom. Available: https://ru.wikipedia.org/wiki/\%D1\%EE $\%$ E5\%E 4\%E8\%ED $\%$ E5\%ED $\%$ E8\%E5 \% $\% 1 \%$ ED $\% \mathrm{E}$ 0\% $2 \% \mathrm{FF} \% \mathrm{E} 3 \% \mathrm{EE} \% \mathrm{EC}$ (Accessed: 02.02.2015)

2. (2015) Neraz'emnye soedineniya. Available: http://www.prikladmeh.ru/lect1.htm (Accessed: 02.02.2015)

3. (2015) Sborochnye soedineniya s natyagom raschet, rekomendacii, metody pressovki. Available: http://teh11.ru/news/sborochnye_soedinenija_s_natjago $\mathrm{m}$ raschet rekomendacii metody pressovki/20 13-04-18-28 (Accessed: 02.02.2015).

4. Qasim H. Shah, Hasan M. Abid (2011) From LS-PREPOST to LS-DYNA: an introduction. LAP LAMBERT Academic Publishing, 132.

5. Brian Gladman (2007) LS-DYNA: keyword user's manual. Volume I. Livermore Software Technology Corporation (LSTC), 2206.

6. (2015) Kriteriy: maksimalnoe napryajenie von Mises. Available: http://help.solidworks.com/2010/russian/Solid
Works/cworks/LegacyHelp/Simulation/Checkin g stresses/prot vonm.html (Accessed: 02.02.2015).

7. (2015) Principal Strains \& Invariants. Available:

http://continuummechanics.org/cm/principalstra in.html (Accessed: 02.02.2015).

8. (2015) What is the resultant acceleration? Available:

http://www.answers.com/Q/What is the result ant acceleration (Accessed: 02.02.2015).

9. (2015) Displacement. Available: http://physics.tutorvista.com/motion/displaceme nt.html\#resultant-displacement (Accessed: 02.02.2015).

10. (2015) Napryajeniya. Available: http://sopromat.in.ua/textbook/stress (Accessed: 02.02.2015).

11. (2015) How to find resultant velocity. Available:

http://www.ehow.com/how 8263993 resultantvelocity.html (Accessed: 02.02.2015). 